\title{
Stress Response Signaling
}

National Cancer Institute

\section{Source}

National Cancer Institute. Stress Response Signaling. NCI Thesaurus. Code C19944.

Any intracellular process that involves a chemical or mechanical stimulus and results in a cellular response that is specific to the stress. 